\title{
Sharp decay rates for the fastest conservative diffusions
}

\section{Vitesse de convergence optimale pour les diffusions nonlinéaires conservatives les plus rapides}

\author{
Yong Jung Kim ${ }^{\mathrm{a}}$ and Robert J. McCann ${ }^{\mathrm{b}}$ \\ a Division of Applied Mathematics, KAIST, Gusong-dong 373-1, Yusong-gu, Taejon, 305-701 South Korea \\ ${ }^{\mathrm{b}}$ Department of Mathematics, University of Toronto, 100 St. George Street, Toronto, ON, M5S 3G3, Canada
}

\begin{abstract}
In many diffusive settings, initial disturbances will gradually disappear and all but their crudest features — such as size and location - will eventually be forgotten. Quantifying the rate at which this information is lost is sometimes a question of central interest. Here this rate is addressed for the fastest conservative nonlinearities in the singular diffusion equation

$$
u_{t}=\Delta\left(u^{m}\right), \quad(n-2)_{+} / n<m \leq n /(n+2), \quad u, t \geq 0, \quad \mathbf{x} \in \mathbf{R}^{n},
$$

which governs the decay of any integrable, compactly supported initial density towards a characteristically spreading self-similar profile. A potential theoretic comparison technique is outlined below which establishes the sharp $1 / t$ conjectured power law rate of decay uniformly in relative error, and in weaker norms such as $L^{1}\left(\mathbf{R}^{n}\right)$.

Dans les milieux dissipatifs, les perturbations initiales disparaissent progressivement, et seuls sont preservés leurs traits les plus grossiers, comme leur taille et leur position. Estimer précisément la vitesse de cette 《disparition》 est parfois une question d'un interêt primordial. Ici, nous donnons cette vitesse pour les diffusions nonlinéaires les plus rapides qui préservent la masse, pour le modèle (1) qui gouverne la diffusion d'une densité initiale, intégrable et à support compact, vers un profil autosimilaire. Pour cela, nous établissons une théorie de comparaison des potentiels, ce qui permet de montrer que la vitesse précise de décroissance est en $1 / t$ pour la norme $L^{1}\left(\mathbf{R}^{n}\right)$, et en fait uniforme pour l'erreur relative.
\end{abstract}

\footnotetext{
‡ The authors are grateful to José Antonio Carrillo and Dejan Slepčev for fruitful discussions, and to Agnes Tourin, Dario Cordero-Erausquin and Emmanuelle Servat for patient help with the version française. We thank the Fields Institute, and the Universities of Toronto, Marne-la-Vallée, and California at Los Angeles and Riverside, where parts of this work were performed, and many colleagues for the stimulating milieu which they helped to create. Partial support of our research by Korea Science \& Engineering Foundation Grant R11-2002-103, United States National Science Foundation Grant DMS 0354729, and Natural Sciences and Engineering Research Council of Canada Grant 217006-03 RGPIN is gratefully acknowledged.

Email addresses: ykim@amath.kaist.ac.kr (Yong Jung Kim), mccann@math.toronto.edu (Robert J. McCann).
} 


\section{Version française abrégée}

Etant donné $p<0$, l'équation des milieux poreux

$$
\left.\frac{\partial u}{\partial t}=\Delta\left(u^{\frac{n+p-2}{n+p}}\right), \quad(\mathbf{x}, t) \in \mathbf{R}^{n} \times\right] 0, \infty[,
$$

a été proposée comme modèle de transport de la chaleur, de diffusion de population, d'infiltration de fluide, d'écoulement par courbure, et d'avalanche de sable; voir Vázquez [17] [18]. Ici, nous nous intéressons au régime de diffusion rapide $p>-n \neq-1$, où localement, le coeffiecient de diffusion $u^{-2 /(n+p)}(\mathbf{x}, t)$ est une fonction décroissante de la densité $u(\mathbf{x}, t) \geq 0$, la quantité qui diffuse. Pour $p \notin[-n, 0[$, Bénilan \& Crandall [3] ont démontré que la masse (et le signe) de la solution sont préservés au cours de l'évolution. Une dilatation de la donnée intégrable $u_{0}(\mathbf{x})=u(\mathbf{x}, 0) \geq 0$ donne la normalisation $1=\int_{\mathbf{R}^{n}} u(\mathbf{x}, t) d \mathbf{x}$. Au cours du temps, la distance entre deux solutions données est une fonction décroissante : plus précisément, Friedman \& Kamin [8] et Vázquez [18] ont démontré (7) pour $p>0$. Si $|p|>n \geq 2$ (ou bien $p=n>2$ ), Dolbeault \& del Pino [7] et Otto [15] ont prouvé que la différence entre deux solutions au sens $L^{1}\left(\mathbf{R}^{n}\right)$ décroit à la vitesse $O\left(t^{-\frac{1}{2}\left(1+\frac{n}{p}\right)}\right)$ quand $t \rightarrow \infty$, si $u_{0}$ et $\tilde{u}_{0}$ sont à variance finie. (Voir aussi Carrillo et al [4]. Lederman \& Markowich [13] ont démontré qu'il suffît que l'entropie relative soit finie quand $p=n=2$.) Si $\tilde{u}_{0}$ est une translation de $u_{0}$, il est impossible de trouver une vitesse plus rapide. Etant donné $p \in] 0, n]$, l'ordre de la convergence en norme $L^{1}$ est $O\left(1 / t^{1 / 2}\right)$, d'après les résultats de Carrillo \& Vázquez [5]. De plus, si les données $\tilde{u}_{0}$ et $u_{0}$ sont à symétrie sphérique, ils ont montré une vitesse de convergence plus rapide, de l'ordre $O(1 / t)$ au sens uniforme pour l'erreur relative (8). Si $p>0$, la décroissance (sans estimation de la vitesse) a déjà été établie pour $u_{0}, \tilde{u}_{0} \in L^{1}\left(\mathbf{R}^{n}\right)$ par Vázquez [18]. Une translation en temps $\tilde{u}_{0}(\cdot)=u(\cdot, 1)$ démontre que la valeur de l'exposant $1 / t$ est optimale; c'est la même valeur que celle découverte par Dolbeault \& del Pino et Otto au point de transition $p=n$. Dans les résultats énoncés ci-dessous, nous obtenons la vitesse optimale $O(1 / t)$ au sens plus fort (8), sans hypothèse de symétrie, dans le régime le plus rapide $p \in] 0,2]$ des diffusions nonlinéaires conservatives. L'hypothèse sur l'entropie relative est remplacée par (11)-(14). Des résultats dans cette direction ont été conjecturés par Carrillo \& Vázquez, et aussi par Denzler \& McCann [6]. Le cas $p=0$ étudié par Galaktionov, Peletier, \& Vázquez [9] est très différent. Pour la dimension spatiale $n=1$, on peut trouver un résultat comparable si $p \in] 1, \infty[$. Dans ce cas aussi on a $n+p>2$, ce qui entraine la positivité de l'exposant $m:=1-2 /(n+p)$, une condition nécessaire pour la parabolicité de l'évolution (2).

Malheureusement, on ne peut pas utiliser le principe du maximum pour (2) afin de comparer $\tilde{u}$ et $u$ directement, parce que les deux solutions ont la même masse : l'inégalité globale $\tilde{u}(\cdot, t) \geq u(\cdot, t)$ est impossible, sauf dans le cas trivial où $\tilde{u}=u$. Au contraire, nous proposons de considérer l'évolution des potentiels Newtoniens $U=\phi * u$ et $\tilde{U}=\phi * \tilde{u}$, obtenus par convolution des densités $u(\cdot, t)$ et $\tilde{u}(\cdot, t)$ à chaque instant, avec la solution fondamentale (9) de l'équation de Laplace.

Formellement, l'application de l'opérateur $\Delta^{-1}:=\phi *$ à l'évolution (2) donne

$$
\frac{\partial U}{\partial t}=u^{m}>0
$$

Donc nous pouvons penser que $U(\mathbf{x}, t)$ croit vers zero $(n \geq 3)$ ou vers $+\infty(n \leq 2)$. En fait, nous allons démontrer que

$$
\tilde{U}(\cdot, t-T) \leq U(\cdot, t) \leq \tilde{U}(\cdot, t+S)
$$

si $T<t=t_{0}<S$ sont suffisamment grandes (pour la donnée initiale). Etant donné que la difference $V=U-\tilde{U}$ satisfait une équation linéaire et parabolique

$$
\frac{\partial V}{\partial t}=a(\mathbf{x}, t) \Delta V, \quad a(\mathbf{x}, t)=\frac{u^{m}-\tilde{u}^{m}}{u-\tilde{u}} \geq 0
$$


les inégalités (4) sont préservé pour tout temps $t \geq t_{0}$. Si nous pouvons estimer précisément la décroissance de

$$
\tilde{U}(\cdot, t+S)-\tilde{U}(\cdot, t-T)=o(1) \quad \text { quand } \quad t \rightarrow \infty
$$

entre les deux translatées en temps d'une solution donnée, comme la solution $\tilde{u}(\mathbf{x}, t)=t^{-n \alpha} \hat{\rho}\left(\mathbf{x} / t^{\alpha}\right)$ de Barenblatt (10), alors (4) entrainera que la différence $V(\cdot, t)$ décroit à la même vitesse. Finalement, les propriétés de régularisation de la diffusion permettent de préciser la vitesse de convergence (8) de $\Delta V=u-\tilde{u}$.

\section{Main text}

For $p<0$, the nonlinear diffusion equation (2) reviewed by Vázquez [17] [18] has been proposed as a model for heat transport, fluid seepage, population spreading, curvature flow, and avalanches in sandpiles. Here instead we are interested in the singular or fast diffusion regime $p>-n \neq-1$, where the local diffusivity $u^{-2 /(n+p)}(\mathbf{x}, t)$ depends inversely on the concentration $u(\mathbf{x}, t) \geq 0$ of the scalar diffusing. For $p \notin[-n, 0[$, Bénilan \& Crandall [3] showed the equation preserves mass (and sign), so a suitable scaling normalizes integrable initial data $u_{0}(\mathbf{x})=u(\mathbf{x}, 0) \geq 0$ to yield $1=\int_{\mathbf{R}^{n}} u(\mathbf{x}, t) d \mathbf{x}$. Any two such solutions become more and more similar as time progresses: Friedman \& Kamin [8] and Vázquez [18] showed

$$
\|u(\cdot, t)-\tilde{u}(\cdot, t)\|_{L^{1}\left(\mathbf{R}^{n}\right)}=o(1) \quad \text { as } \quad t \rightarrow \infty .
$$

When $|p|>n \geq 2$ (and when $p=n>2$ ), Dolbeault \& del Pino [7] and Otto [15] proved the norm in (7) disappears at rate $O\left(t^{-\frac{1}{2}\left(1+\frac{n}{p}\right)}\right)$ if $u_{0}$ and $\tilde{u}_{0}$ have finite variance. (See also Carrillo et al [4]; finite relative entropy suffices when $p=n=2$, from Lederman \& Markowich [13].) Taking $\tilde{u}_{0}$ to be a translate of $u_{0}$ shows that this rate cannot be improved. For $p \in] 0, n]$, the norm (7) was shown to disappear at rate $O\left(1 / t^{1 / 2}\right)$ by Carrillo \& Vázquez [5]. Moreover, for radially symmetric $\tilde{u}_{0}$ and $u_{0}$, they gave a smaller bound $O(1 / t)$ uniformly on the relative error

$$
\left\|\frac{u(\mathbf{x}, t)}{\tilde{u}(\mathbf{x}, t)}-1\right\|_{L^{\infty}\left(\mathbf{R}^{n}\right)}=o(1) \quad \text { as } \quad t \rightarrow \infty,
$$

which had been shown to converge (without a rate) for $p>0$ and $u_{0}, \tilde{u}_{0} \in L^{1}\left(\mathbf{R}^{n}\right)$ by Vázquez [18]. A time translation $\tilde{u}_{0}(\cdot)=u(\cdot, 1)$ shows the exponent $1 / t$ cannot be improved, and it agrees with the rate found by Dolbeault \& del Pino and Otto at the transition point $p=n$. In the work announced below, we prove the sharp $O(1 / t)$ rate holds for the stronger norm (8) without the assumption of radial symmetry in the fastest range $p \in] 0,2]$ of conservative nonlinearities. The finite relative entropy hypothesis is replaced by (11)-(14). A result in this vein was conjectured by Carrillo \& Vázquez, as well as Denzler \& McCann [6]. The case $p=0$ investigated by Galaktionov, Peletier, \& Vázquez [9] is quite different. In one space dimension, $n=1$, an analogous result can be proved assuming $p \in] 1, \infty[$. Since $n+p>2$, we have positivity of the power $m:=1-2 /(n+p)$ in all dimensions, a necessary condition for the forward evolution in time (2) to be parabolic as written.

The maximum principle for (2) cannot be used to compare $\tilde{u}$ to $u$ directly since both solutions have the same mass: domination $\tilde{u}(\cdot, t) \geq u(\cdot, t)$ never occurs except in the trivial case $\tilde{u}=u$. Instead, under suitable hypotheses, we propose to consider the evolution of the Newtonian potentials $U=\phi * u$ and $\tilde{U}=\phi * \tilde{u}$ obtained by convolving the densities $u(\cdot, t)$ and $\tilde{u}(\cdot, t)$ at each instant in time with the Green's function of the Laplacian: 


$$
\phi(\mathbf{x}):=-|\mathbf{x}|^{2-n} / c_{n} \text { with } c_{n}:=(n-2) \omega_{n},
$$

where $\omega_{n}:=2 \pi^{n / 2} / \Gamma(n / 2)$ is the surface area of the unit sphere in $\mathbf{R}^{n}, n \geq 3$. We may also take $\phi(\mathbf{x}):=(2 \pi)^{-1} \ln |\mathbf{x}|$ for $n=2$ or $\phi(\mathbf{x}):=|\mathbf{x}| / 2$ for $n=1$.

Formally, applying $\Delta^{-1}:=\phi *$ to the evolution equation (2) yields (3), so we expect $U(\mathbf{x}, t)$ to increase monotonically to zero $(n \geq 3)$ or to $+\infty(n \leq 2)$. In fact, we shall eventually show $U(\cdot, t)$ to be sandwiched (4) between advanced and retarded copies of $\tilde{U}(\cdot, t)$ for fixed $T<t=t_{0}<S$ large enough (depending on the initial data). Since the difference $V=U-\tilde{U}$ satsifies a linear parabolic equation (5), this sandwiching (4) extends to all $t \geq t_{0}$. If we now quantify the decay (6) of the discrepancy between two time-delayed copies of any single solution $\tilde{U},(4)$ implies the same rate of decay for the difference $V(\cdot, t)$. The smoothing properties of the equation then allow us to quantify a rate of convergence in (8) for $\Delta V=u-\tilde{u}$.

One explicit evolution $\tilde{u}=\rho$ is known. Discovered by Barenblatt [2], Pattle [16], Zel'dovich \& Kompaneets [19], it spreads self-similarly $\rho(\mathbf{x}, t)=t^{-n \alpha} \hat{\rho}\left(\mathbf{x} / t^{\alpha}\right)$ at rate $\alpha:=\frac{1}{2}\left(1+\frac{n}{p}\right)$ from a point mass at time zero, with a spatial profile given by

$$
\hat{\rho}(\mathbf{x}):=\left(B|\mathbf{x}|^{2}+A\right)_{+}^{-(n+p) / 2} .
$$

Here $(\lambda)_{+}:=\max \{\lambda, 0\},(2 p B)^{-1}+2(n+p)^{-1}=1$, and $A \in \mathbf{R}$ is arbitrary, but is made fixed and positive by the normalization $1=\int_{\mathbf{R}^{n}} \hat{\rho}(\mathbf{x}) d \mathbf{x}$. For $p>0$, it is not hard to check that $R:=\phi * \rho$ satisfies $\|R(\cdot, t+S)-R(\cdot, t-T)\|_{L^{\infty}\left(\mathbf{R}^{n}\right)}=O\left(t^{-\frac{n}{2 p}(n+p-2)}\right)$, and $\|\rho(\cdot, t+S) / \rho(\cdot, t-T)-1\|_{L^{\infty}\left(\mathbf{R}^{n}\right)}=O(1 / t)$ as $t \rightarrow \infty$.

It remains to specify the precise hypotheses [11] under which we have carried out the argument indicated above. Fix $p>0$ and $n \geq 2$. Following Aronson \& Bénilan [1] and Herrero \& Pierre [10], a solution will refer to a smooth function $u(\mathbf{x}, t)>0$ satisfying (2) on the open halfspace, for which there exists an initial profile $0 \leq u_{0} \in L^{1}\left(\mathbf{R}^{n}\right)$ such that

$$
0=\lim _{t \downarrow 0}\left\|u(\cdot, t)-u_{0}(\cdot)\right\|_{L^{1}\left(\mathbf{R}^{n}\right)}
$$

The age of the profile can be inferred from the thickness of its tails; indeed, given $\tau=0$ [14] or $\tau>0$ [5], Lee \& Vázquez and Carrillo \& Vázquez showed $\lim _{|\mathbf{x}| \rightarrow \infty} B|\mathbf{x}|^{2} u^{2 /(n+p)}(\mathbf{x}, t)=\tau+t$ holds for all $t>0$ if it holds when $t=0$. Let us therefore assume the limit

$$
\tau:=\lim _{|\mathbf{x}| \rightarrow \infty} B|\mathbf{x}|^{2} u_{0}^{2 /(n+p)}(\mathbf{x})
$$

exists, and that the Barenblatt profile of the same age $\rho(\cdot, \tau)$ shares all moments of $u_{0}(\cdot)$ up to order $p$ :

$$
0=\int x_{1}^{\beta_{1}} \cdots x_{n}^{\beta_{n}}\left[u_{0}(\mathbf{x})-\rho(\mathbf{x}, \tau)\right] d \mathbf{x}, \quad \text { whenever }|\beta|<p
$$

for each multi-index $\beta \in \mathbf{N}^{n}$ with $|\beta|:=\sum_{i=1}^{n} \beta_{i} \geq 0$. Here $\rho(\mathbf{x}, 0):=\delta(\mathbf{x})$ denotes the Dirac mass. For $p \in] 0,2]$, this restriction costs no generality, since translation and scaling normalize mass and center of mass. To state our main result, we assume furthermore that $p$-th moment of the difference converges:

$$
\int|\mathbf{x}|^{p}\left|u_{0}(\mathbf{x})-\rho(\mathbf{x}, \tau)\right| d \mathbf{x}<\infty .
$$

Theorem 1 (Sharp $L^{\infty}$ convergence rate for relative error) Fix $p>0$ and $n \geq 2$. Let $u(\mathbf{x}, t) \geq 0$ be a solution to (2) satisfying the initial conditions (11)-(14). Then

$$
C_{r e l}\left(p, u_{0}\right):=\limsup _{t \rightarrow \infty} t\left\|\frac{u(\cdot, t)}{\rho(\cdot, t)}-1\right\|_{L^{\infty}\left(\mathbf{R}^{n}\right)}<+\infty .
$$


The same sharp $O(1 / t)$ rate of convergence in $L^{1}$ follows immediately:

$$
\limsup _{t \rightarrow \infty} t\|u(\cdot, t)-\rho(\cdot, t)\|_{L^{1}\left(\mathbf{R}^{n}\right)} \leq\left\|u_{0}\right\|_{L^{1}\left(\mathbf{R}^{n}\right)} C_{r e l}\left(p, u_{0}\right) .
$$

When $p \in] 1, \infty[$ and $n=1$, the same conclusions hold, and we may drop hypotheses (13)-(14) as long as the mass and center of mass of $u_{0}$ and $\rho(\mathbf{x}, \tau)$ coincide.

The proof of Theorem 1 relies on ideas sketched out above, plus two key lemmas to establish (4). Many technical devices are required to knit these ideas together, including finite time barriers for the long tails of $u(\mathbf{x}, t)$ constructed by comparison with the infinite mass $A \leq 0$ self-similar solutions (10), starting from (12). The first key lemma states that if enough moments vanish, the Newtonian potential $V=\Delta^{-1} v$ will decay two powers of $|\mathbf{x}| \rightarrow \infty$ slower than its charge density $v(\mathbf{x})$ :

Lemma 2 (Spatial decay of Newtonian potential) Fix $\lambda, L, p>0$ positive. Let $V=\phi * v$ denote the Newtonian potential of a signed Radon measure $v(\mathbf{y})$ on $\mathbf{R}^{n}$, whose density satisfies

$$
\begin{aligned}
& |\mathbf{y}|^{n+p}|v(\mathbf{y})|<L \quad \text { if }|\mathbf{y}|>\lambda, \quad M:=\int|\mathbf{y}|^{p}|v(\mathbf{y})| d \mathbf{y}<\infty, \quad \text { and } \\
& 0=\int \mathbf{y}^{\beta} v(\mathbf{y}) d \mathbf{y} \quad \text { for each } \beta \in \mathbf{N}^{n} \text { of degree }|\beta| \in[0, p[.
\end{aligned}
$$

If $p \geq(2-n)_{+}$there exist a constant $C_{p}=C(n, p)<\infty$ such that

$$
|\mathbf{x}|^{n+p-2}|V(\mathbf{x})| \leq(M+(n-1) L) C_{p} \quad \text { when }|\mathbf{x}|>3 \lambda
$$

The proof of this lemma starts from $V(\mathbf{x})=\int_{\mathbf{R}^{n}} \phi(\mathbf{x}-\mathbf{y}) v(\mathbf{y}) d \mathbf{y}$, and relies on binomial expansion of the Green's function $\phi(\mathbf{x}-\mathbf{y})=\phi(\mathbf{x})(1-\epsilon)^{1-\frac{n}{2}}$ in $\epsilon=\left(2 \mathbf{x} \cdot \mathbf{y}-|\mathbf{y}|^{2}\right) /|\mathbf{x}|^{2}$. The vanishing moments (17) ensure all powers of $|\mathbf{x}|^{-1} \mathbf{y}$ less than $p$ integrate to zero, while the bounds (16) and Taylor's remainder control the coefficient of $\phi(\mathbf{x}) /|\mathbf{x}|^{p}$ in the terms that persist.

The second lemma is a "tortoise and hare" type result, which asserts that a large enough headstart enables the Newtonian potential of any solution to overtake its competitors, if their densities share enough moments initially.

Lemma 3 (Newtonian potentials leap-frog) Fix $n \geq 1$ and $p>(2-n)_{+}$. Let $U=\phi * u$ and $\tilde{U}=\phi * \tilde{u}$ be the Newtonian potentials at each instant in time, of two solutions $u(\mathbf{x}, t)$ and $\tilde{u}(\mathbf{x}, t)$ to (2) and (12), whose initial difference satisfies the decay condition $\limsup _{\mathbf{x} \rightarrow \infty}|\mathbf{x}|^{n+p-2}|U(\mathbf{x}, 0)-\tilde{U}(\mathbf{x}, 0)|<\infty$.

Given $T_{0}>0$, taking $T>0$ large enough ensures

$$
U(\mathbf{x}, t) \geq \tilde{U}\left(\mathbf{x}, T_{0}\right) \text { for all } t \geq T \text { and } \mathbf{x} \in \mathbf{R}^{n} .
$$

The content of this lemma is all in the tails, since it is enough to obtain (19) for large $\mathbf{x}$. Its proof begins with (3) which gives $U(\mathbf{x}, t)-U(\mathbf{x}, 0)=\int_{0}^{t} u^{m}(\mathbf{x}, s) d s$. Vázquez' result (8) bounds this integrand above and below by multiples of

$$
\rho^{m}(\mathbf{x}, s)=\left(B s^{-1}|\mathbf{x}|^{2}+A s^{n / p}\right)^{(2-n-p) / 2} .
$$

For large $\mathbf{x}$, the $A$ term can be absorbed into the $B$ term, whose tails decay similarly to the hypothesized difference $|U(\mathbf{x}, 0)-\tilde{U}(\mathbf{x}, 0)|$. Selecting the length of time $t$ that we integrate over now changes the coefficient in front of these tails by as much or little as we please.

Under the assumptions of Theorem 1, these two lemmas allow us to deduce (4) for large enough times $0<T<t_{0}<S$. From $R(\mathbf{x}, t-T) \leq U(\mathbf{x}, t) \leq R(\mathbf{x}, t+S)$ on $(\mathbf{x}, t) \in \mathbf{R}^{n} \times\left[t_{0}, \infty[\right.$ we get $\| U(\cdot, t)-$ $R(\cdot, t) \|_{L^{\infty}\left(\mathbf{R}^{n}\right)}=O\left(t^{-\frac{n}{2 p}(n+p-2)}\right)$. To arrive at (15) from here is a lengthy but satisfying process, combining scaling properties of the evolution with a priori smoothness estimates of Ladyženskaja, Solonnikov, and Ural'ceva [12] in the bulk, and barriers to control the tails, as in the radial argument of Carrillo \& Vázquez 
[5]. The details of this process and other developments sketched out above appear in a forthcoming publication [11].

\section{References}

[1] D.G. Aronson \& P. BÉnilan. Regularite des solutions de l'equation des milieux poreux dans $R^{N}$. $C$. $R$. Acad. Sci. Paris Ser. A-B 288 (1979) A103-A105.

[2] G.I. Barenblatt. On some unsteady motions of a liquid or gas in a porous medium. Akad. Nauk. SSSR. Prikl. Mat. Mekh. 16 (1952) 67-78.

[3] P. Bénilan \& M.G. Crandall. The continuous dependence on $\varphi$ of solutions of $u_{t}-\Delta \varphi(u)=0$. Indiana Univ. Math. J. 30 (1981) 161-177.

[4] J.A. Carrillo, A. Jüngel, P.A. Markowich, G. Toscani \& A. Unterreiter. Entropy dissipation methods for degenerate parabolic problems and generalized Sobolev inequalities. Monatsh. Math. 133 (2001) 1-82.

[5] J.A. Carrillo \& J.L. VÁzquez. Fine asymptotics for fast diffusion equations. Comm. Partial Differential Equations 28 (2003) 1023-1056.

[6] J. Denzler \& R.J. MCCANn. Fast diffusion to self-similarity: complete spectrum, long time asymptotics, and numerology. Arch. Rational Mech. Anal. 175 (2005) 301-342.

[7] J. Dolbeault \& M. Del Pino. Best constants for Gagliardo-Nirenberg inequalities and applications to nonlinear diffusions, J. Math. Pures Appl. 81 (2002) 847-875.

[8] A. Friedman \& S. Kamin. The asymptotic behaviour of a gas in an $n$-dimensional porous medium. Trans. Amer. Math. Soc. 262 (1980) 551-563.

[9] V.A. Galaktionov, L.A. Peletier \& J.L. VÁzquez. Asymptotics of the fast-diffusion equation with critical exponent. SIAM J. Math. Anal. 31 (2000) 1157-1174.

[10] M.A. Herrero \& M. Pierre. The Cauchy problem for $u_{t}=\Delta u^{m}$ when $0<m<1$. Trans. Amer. Math. Soc. 291 (1985) 145-158.

[11] Y.-J. Kim \& R.J. MCCAnn. Potential theory and optimal convergences rates in fast nonlinear diffusion. Preprint \#23 at www.math.toronto.edu/ mccann

[12] O.A. LadyžEnskaja, V.A. Solonnikov \& N. N. URAL'CEvA. Linear and Quasilinear Equations of Parabolic Type (Russian). Translated from the Russian by S. Smith. Translations of Mathematical Monographs 23. American Mathematical Society, Providence RI, 1967.

[13] C. Lederman \& P.A. Markowich. On fast-diffusion equations with infinite equilibrium entropy and finite equilibrium mass. Comm. Partial Differential Equations 28 (2001) 301-332.

[14] K.-A. LeE \& J.L. VÁzQUez. Geometrical properties of solutions of the porous medium equation for large times. Indiana Univ. Math. J. 52 (2003) 991-1016.

[15] F. Отто. The geometry of dissipative evolution equations: the porous medium equation Comm. Partial Differential Equations 26 (2001) 101-174.

[16] R.E. PATTLE. Diffusion from an instantaneous point source with concentration dependent coefficient. Quart. J. Mech. Appl. Math. 12 (1959) 407-409.

[17] J.L. VÁzQuez. "An Introduction to the mathematical theory of the Porous Medium Equation" in Shape Optimization and Free Boundaries (Montreal, PQ, 1990), 347-389. NATO Adv. Sci. Inst. Ser. C Math. Phys. Sci. 380 Kluwer Acad. Publ., Dordrecht, 1992

[18] J.L. VÁzquEz. Asymptotic behaviour for the porous medium equation posed in the whole space. J. Evol. Equ. 3 (2003) $67-118$.

[19] Ya.B. Zel'dovich AND A.S. Kompaneets. Towards a theory of heat conduction with thermal conductivity depending on temperature. In Collection of Papers dedicated to 70th Anniversary of A.F. Ioffe, 61-72, Izd. Akad. Nauk. SSSR, Moscow, 1950. 